\title{
Nutraceuptides: Perspectives into the Future of Supplementary Food Sciences and Therapeutic Technology
}

\author{
Andrew G Mtewa* \\ Chemistry Section, Institute of Technology, Malawi University of Science and Technology, Thyolo, Malawi \\ Department of Pharmacology and Therapeutics, School of Medicine, Uganda
}

*Corresponding author: Andrew G Mtewa, Malawi University of Science and Technology, Malawi, P.O. Box 5196, Limbe.
Received Date: November 22, 2018

Published Date: December 06, 2018

Keywords: Nutraceuticals; Nutraceuptides; Venom therapy; Animal peptides; Pharmaceutics; Health management

\section{Perspective}

Animal venom peptides, pharmaceuticals and nutrients from natural products are currently playing a significant role in both preventing and managing health and its complications worldwide. The term "Nutraceuticals" was first used after being derived from a combination of "Nutrition" and "Pharmaceuticals" by Dr. Stephen De Felice in 1989. They are plant sources containing chemicals that are primarily sought for their nutritive value but also contain significant health properties (Sarin, Manisha, Singh, \& Kumar, 2012). These properties could be potential antioxidant activities, immunomodulation and other important pharmacological activities against disease pathways (Park, Wang, Kim, \& Zhong-Ji). Besides home benefits, nutraceuticals from natural product have revolutionized the pharmaceutical and food industries by demonstrating potential of replacing and/or improving synthetic drugs and supplements like anti-oxidants and vitamin among others. As the combination of nutrients and pharmaceuticals from plant sources brought the science community to the field of nutraceuticals, a careful combination of animal venom peptides to nutraceutical research will potentially lead to therapeutic and supplementary products that will be more pharmacovigilant with chemically stable and bioavailable molecules in the prevention and management of current and/or future health complications.

A review of various health and disease management approaches using natural products showed promising positive results if nutraceuptides were solely studied as a unit and taken up as a new model in drug discovery research. A good source example of animal naturally with both nutritive and pharmaceutical peptides is Silkworm. Silk worm extracts and its droppings are reported to have natural special benefits as dietary supplements for patients with circulatory disorders and heart disease where they act by the reduction of serum cholesterol and dissolve vascular plaque [1-3]. In Asia, it is reported to be mixed with saw palmetto, ginseng and $\mathrm{Ma}$ Huang to promote male potency just as termite extracts combined with mustard oil have also shown potential in some Ayurvedic practices $[4,5]$. This combination can better be studied if looked at as a separate field called "nutraceuptides". I am therefore defining nutraceuptides as supplementary food products made from a combination of both plant and animal peptide bioactive in the prevention, treatment and/or management of health complications. In our proposition, I believe that with the current scientific evidence and community current community use, specialties can be developed in this area for the betterment of health and disease management. Challenges that I envisage in this special field of study include management of peptides which can easily get denatured in room temperature as opposed to herbal extracts or compounds which largely survive in the same conditions. This challenge can be overcome through careful optimization storage and handling of animal extracts and peptides. One opportunity to be got from this area is the perfection of skills on animal venom and peptide extraction and management which can easily be adapted from traditional practices and improved. For a functional pedagogical 
system with the proposed almagation, there is need now to start working towards this significant shift in learning and studying drug discovery and disease management with nutraceuptides as a potentially better approach in the offing. In a few years' time, it will be easier to formalize the inclusion of this approach in training curricula as well as standard laboratory and research protocols.

\section{Acknowledgement}

None.

\section{Conflict of Interest}

No Conflict of Interest.

\section{References}

1. Chou TY, Yang MJ, Tseng SK, Lee SS, Chang CC (2018) Tea silkworm droppings as an enriched source of tea flavonoids. Journal of food and drug analysis 26(1): 41-46.

2. Kunz RI, Brancalhao RMC, Ribeiro LFC, Natali MRM (2016) Silkworm Sericin: Properties and Biomedical Applications. BioMed Research International 2016: 8175701.

3. Park P, Wang TT, Kim E, Zhong Ji Q (2016) Nutraceuticals. Recent Advances of Bioactive Food Components. Journal of Chemistry 2016: 5874726 .

4. Sarin R, Manisha S, Singh R, Kumar S (2012) Nutraceuticals: A review. International Research Journal of Pharmacy 3(4): 95-99.

5. Turk A (2012) 10 Surprising Uses of Insects in Medicine. Nursing School Rankings. 